\title{
Millennials y redes sociales: estrategias para una comunicación de marca efectiva
}

\author{
Dr. José Javier Ruiz Cartagena | josejavier.ruiz@protocoloimep.com \\ Instituto Mediterráneo Estudios de Protocolo
}

\section{Palabras clave}

Generación Milenial, Redes Sociales, marca, marketing, comunicación, marketing.

\section{Sumario}

1. Introducción 1.1. Delimitación de la Generación Milenial. 1.2. Caracterización de la Generación Milenial. 1.3. Claves para una estrategia de marca efectiva dirigida a Milenials en Redes Sociales. 2. Metodología. 3. Resultados y discusión.4.

Conclusiones. 5. Bibliografía

\section{Resumen}

En el artículo se recopilan las principales claves de comunicación para generar estrategias de marca efectivas dirigidas a la Generación Millennials a través de las Redes Sociales. Se trata de una construcción del estado del arte, una metodología de investigación cualitativodocumental de carácter críticointerpretativa que pretende proporcionar al lector una puesta al día sobre conceptos útiles en el área de marca, Redes Sociales y generaciones digitales. En primer lugar, se ha delimitado a la Generación Millennial como aquella formada por personas nacidas entre los años 1981 y 2000. En segundo lugar, se han sintetizado los principales atributos que definen a esta generación: alta exposición a la tecnología y la información, uso intensivo de Redes Sociales, comportamiento multiplataforma y multitarea, necesidad de socialización, empoderamiento, baja permeabilidad a los medios tradicionales, carácter exigente frente a las marcas, rearme ético y personalidad individualista. Por último, se han sintetizado las principales claves de comunicación para generar estrategias de marca efectivas en Redes Sociales: comunicación multiplataforma, lenguaje empático, contenido relevante, autenticidad y honestidad, uso del boca a boca, comunicación alineada con los valores de la generación, valores superiores de marca, participación activa, contenidos en un formato audiovisual y estrategia de recompensas.

\section{Cómo citar este texto:}

José Javier Ruiz Cartagena (2017): "Millennials y redes sociales: estrategias para una comunicación de marca efectiva”, en Miguel Hernández Communication Journal, nº 8 , pp. 347 a 367. Universidad Miguel Hernández, UMH (Elche-Alicante). Recuperado el __ de de 20_ de: @ink del artículo en mhjournal.org] 


\title{
Millennials and Social Networks: Strategies for Effective Brand Communication
}

\author{
Dr. José Javier Ruiz Cartagena | josejavier.ruiz@protocoloimep.com \\ Instituto Mediterráneo Estudios de Protocolo
}

\author{
Keywords \\ Millennial generation, social media, brand, \\ marketing, communication, marketing \\ Summary \\ 1. Introduction 1.1. Delimitation of the Millennial \\ Generation. 1.2. Characterization of the \\ Millennial Generation. 1.3. Keys to an effective \\ branding strategy aimed at Milenials in Social \\ Networks. 2. Methodology. \\ 3. Results and discussion. Conclusions. \\ 5. Bibliography
}

\begin{abstract}
This paper summarizes the key digital marketing brand strategies to reach millennials in social media. The methodology used is a state of the art literature review, a qualitativedocumentary research methodology with a critical interpretative character that reviews the writing production which describes the current state of brand, social media and millennials research. Firstly, millennial generation has been defined as the generation of people born between the early 1980s and the early 2000s.
\end{abstract}

Secondly, the key characteristics that define this generation has been described: intense exposure to technology and information, strong use of social media, multi-platform and multi-task users, intensive socialization, empowerment, traditional media irrelevance, demand of brands more convenient and ethical than ever and individualisim. Finally, the result of the review is in the form of "how to" tips and strategies to improve best practice of organization brand strategies: development of multi-platform communication, bring empathy, design of relevant content, impulse of authenticity and honesty, boost engagement by word-of-mouth, align millennial values and brand communications, encourage active participation, increase brand equity by using corporate responsability, use visual content and set a reward strategy.

\section{How to cite this text:}

José Javier Ruiz Cartagena (2017): "Millennials and Social Networks: Strategies for Effective Brand Communication", en Miguel Hernández Communication Journal, nº, pp. 347 to 367. Universidad Miguel Hernández, UMH (Elche-Alicante). Accessed 20_ in: [paper link in mhjournal.org] 


\section{Introducción}

El objetivo del artículo es recopilar las principales claves de comunicación para generar estrategias de marca efectivas dirigidas a la Generación Milenial a través de las Redes Sociales. Se pretende responder principalmente a tres cuestiones: ¿Cómo queda delimitada actualmente la Generación Milenial?, ¿Cuáles son las principales características de esta generación? ¿Cuáles son las claves para que las organizaciones logren crear marcas que consigan vínculos fuertes con la Generación Milenial en Redes Sociales?. Para ello, se ha realizado una construcción del estado del arte a partir de la información e investigaciones más relevantes sobre el ámbito de la comunicación de marca, Redes Sociales y generaciones digitales. De este modo, se quiere proporcionar al lector una puesta al día sobre estos conceptos. El artículo identifica los aspectos relevantes conocidos, y concluye con unas recomendaciones a las organizaciones para que gestionen de manera adecuada su presencia en las Redes Sociales y consigan un vínculo mayor con su público.

La Generación Milenial genera una gran fascinación tanto en el ámbito empresarial como en el académico (Bolton et al., 2013). Su gran tamaño produce un enorme interés entre los profesionales del marketing, que trabajan por entender su comportamiento (Swinarski, Parente \& Noce, 2010). Este grupo supondrá el 75 por ciento de la fuerza laboral del mundo en el año 2025 (Estudio BBVA, 2015) y constituye ya el principal segmento de consumidores del planeta (Duffet, 2015).

Desde el punto de vista de la comunicación y el marketing, hay una gran oportunidad para que las empresas aprovechen las ventajas de la presencia en Redes Sociales para crear un vínculo con sus clientes (Hudson et al., 2015). Sin embargo, la literatura sobre la Generación Milenial, Redes Sociales y marca sugiere que hay una brecha en el conocimiento de las empresas a la hora de alcanzar a esta generación. Prueba de ello es que muchas organizaciones fallan a la hora de construir relaciones con este público a través de los nuevos canales de comunicación. Para alcanzar con efectividad a los Milenials a través de las Redes Sociales, se debe conocer bien su comportamiento y motivaciones dentro de las mismas (Barton, Koslow \& Beauchamp, 2014). Por este motivo, resulta de interés hacer una recopilación de la numerosa, fragmentada y extensa literatura de los últimos años sobre la Generación Milenial y su forma de interactuar con las marcas en Redes Sociales, más aún cuando se trata de un área de conocimiento en constante y rápida evolución. 


\subsection{Delimitación de la Generación Milenial.}

La Generación Milenial o Generación Y ha sido analizada ampliamente por diversos estudios, y aunque no hay un consenso general sobre el rango de edad de las personas que la componen, la mayoría la definen como la compuesta por los nacidos entre los años 1981 y 2000. En ocasiones se amplían estos límites hasta los años 1977 y 2004, según el autor o el estudio (DeVaney, 2015). Esta generación se enmarca dentro de la clasificación ampliamente aceptada de cohortes que incluye a la Generación Silenciosa (1925-1945), la Generación Baby Boomer (1946-1960), la Generación X (19611981) y la Generación Y o Milenials (Brosdahl \& Carpenter, 2011). Esta clasificación ha incorporado recientemente nuevas generaciones más jóvenes como la Z.

Las denominaciones de la Generación Milenial son variadas: Generación Y, Generación iPod, Generación Global o nativos digitales (Leung, 2003; Prensky, 2001). En cualquier caso, se trata de la primera generación que ha pasado su vida entera en un entorno digital, un hecho que ha condicionado sus valores, su trabajo y la forma de relacionarse con el mundo (Bennett, Maton \& Kervin, 2008; Wesner \& Miller, 2008). Los Milenials son considerados hijos de la Generación Baby Boomer, y los acontecimientos clave de su infancia fueron la globalización, los ataques del 11 de septiembre y la era de Internet. Esta generación está formada por más de 80 millones de personas en Estados Unidos y más de 50 millones en Europa, y en el año 2025 supondrá el 75 por ciento de la fuerza laboral del planeta (Estudio BBVA, 2015).

Los Milenials generan una gran fascinación tanto en el ámbito empresarial como en el académico (Bolton et al., 2013). No en vano, constituyen ya el principal segmento de consumidores del mundo (Duffet, 2015). La Generación $\mathrm{Y}$ tiene un tamaño tres veces mayor que su antecesora, la Generación X, y conforma el mayor mercado de consumo desde la Generación Baby Boomer, de ahí el enorme interés en marketing por conocer el

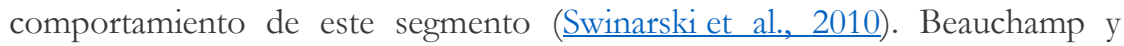
Barnes (2015) consideran a esta generación, por su tamaño y poder de compra, la más importante hoy para su estudio dentro del ámbito del marketing. 


\subsection{Caracterización de la Generación Milenial}

Las características que definen a la Generación Milenial se han estudiado de forma extensa durante los últimos años. Es amplio el reconocimiento de muchos de los atributos que la conforman, como por ejemplo su afinidad por la tecnología. Es una generación que ha crecido paralelamente al surgimiento de las Redes Sociales (Parment, 2011), en una era de comunicación instantánea y saturación de información (Barbagallo, 2003). Sin embargo, algunos autores también advierten del riesgo de estereotipar a esta generación. Smith (2012) y Bolton et al. (2013) consideran que estudiar a los Milenials como un grupo único y homogéneo no favorece el entendimiento de sus necesidades a la hora de establecer estrategias de marketing en las empresas y desarrollar vínculos efectivos con las marcas. Para Smith (2012) muchos estudios no examinan en profundidad esta generación y la implicación de su relación con las Redes Sociales; hay mucha heterogeneidad dentro de la Generación Y, y la extensa literatura sobre ésta ofrece más preguntas que respuestas.

No obstante lo anterior, hay un amplio consenso en la definición de los rasgos generales que definen a la Generación Y, y sobre sus diferencias y cambios de valores con respecto a sus generaciones predecesoras (Hyllegard et al., 2011). Alsop (2008) define a los Milenials como personas más tolerantes, optimistas, inquietas, cívicas, orientadas al trabajo en equipo y concienciadas en la búsqueda de un equilibrio entre el trabajo y el ocio. Syrett y Lammiman (2003) dibujan un perfil individualista, tecnológico, sofisticado, maduro y con un fuerte sentido de identidad. Los propios Milenials se definen como tecnológicos, modernos, atrevidos y rebeldes (Barton, Koslow \& Beauchamp, 2014). Burstein (2013) resalta el entorno de crisis económica en el que crecieron, que ha condicionado muchas de las características de su comportamiento; un perfil propenso al activismo y la protesta, con capacidad para el emprendimiento, pero que también retrasa la emancipación, el matrimonio o la compra de su primera casa. A pesar de la crisis económica, es un grupo que ha crecido en una cultura de consumo, materialista, donde sus vidas hasta ahora han transcurrido en un entorno de prosperidad.

Tras una revisión de la literatura referente a la caracterización de la Generación Milenial, resulta útil hacer una síntesis de la información más relevante. Los principales atributos destacados por diferentes autores en relación a cómo es la Generación Y se detallan a continuación: 
- Alta exposición a la tecnología y la información. Es un rasgo que determina profundamente el comportamiento de esta generación. Los Milenials son "nativos digitales", y usan las Tecnologías de la Información y la Comunicación de forma natural (Prensky, 2011). Quieren estar permanentemente conectados y confían fuertemente en la tecnología para comunicarse con el mundo, dado que han crecido paralelamente con el avance de Internet, las Redes Sociales y la globalización. Los propios Milenials identifican el uso de la tecnología como el elemento que más les diferencia de otras generaciones. Además, tienen la capacidad de hacer varias cosas a la vez, son adictos al móvil y sienten la necesidad de una constante conectividad. Reciben un enorme flujo de información de forma constante, y usan sus dispositivos móviles para casi todo, como desarrollar sus relaciones sociales, encontrar un trabajo o estar informados sobre productos y servicios (Parment, 2011). Para esta generación, es algo natural disponer de toda la información a su alcance, accesible las 24 horas. Cuando buscan información sobre cualquier asunto, Google y las Redes Sociales son las primeras fuentes de información donde consultan (Hershatter \& Epstein, 2010).

- Uso intensivo de la Redes Sociales. Los Milenials son usuarios intensivos de Redes Sociales. Según Statista (2016), hay más de 2.700 millones de personas en el mundo con presencia en Redes Sociales y sólo Facebook cuenta con 1.700 millones de usuarios activos mensuales que ven y generan contenido durante más de 50 minutos al día. La Generación Milenial es la que mayor presencia tiene y la que con mayor intensidad usa esas Redes Sociales. Algunos datos proporcionados por Statista ilustran esa intensidad: el 87 por ciento de los Milenials usan entre dos y tres aparatos electrónicos con conexión a Internet (smartphone, tablet $\mathrm{u}$ ordenador) al menos una vez al día, el 50 por ciento asegura haber usado el smartphone para investigar sobre un producto o servicio durante la compra, el 41 por ciento ha hecho una compra a través del smartphone en alguna ocasión, más del 40 por ciento asegura que compraría todo online si tuviese la oportunidad, el 81 por ciento tiene perfil en Facebook y el 83 por ciento duerme con el móvil.

- Comportamiento multiplataforma y multitarea. La capacidad de cambiar de forma natural de plataforma (móvil, social, PC, videojuegos...) y de hacer varias tareas simultáneamente es una característica de los nativos digitales (Prensky, 2001). Los Milenials utilizan múltiples canales y dispositivos digitales para sus actividades, trabajan en modo multitarea, 
interactúan en Redes Sociales mientras realizan otras tareas simultáneamente, y todo ello de forma más natural que las anteriores generaciones. Como contrapartida, son menos hábiles en la comunicación cara a cara o a la hora de interpretar el lenguaje no verbal en una conversación (Hershatter \& Epstein, 2010).

- Necesidad de socialización y conexión. Los Milenials se caracterizan por una filosofía de participación (Tuten \& Solomon, 2014). De manera natural, usan Internet para generar contenido y expresar sus opiniones. Tienen la necesidad de conexión y de compartir experiencias en los medios sociales, y usan la tecnología y las Redes Sociales para construir y reforzar su identidad y para buscar la aprobación a través de la respuesta de su comunidad a su contenido y opiniones. La necesidad de expresar las opiniones continuamente es claramente consistente con una generación que es más ambiciosa, asertiva y narcisista que generaciones anteriores (Barton, Koslow \& Beauchamp, 2014; Twenge, 2009).

- Empoderamiento. Los Milenials quieren decidir cuándo, dónde y cómo las empresas se comunican con ellos (Parment, 2011). La tecnología les ha otorgado poder frente a empresas y marcas. Constituyen la primera generación que cuenta con acceso en tiempo real a una gran cantidad de información, lo que les permite comprar cuándo y cómo quieren. Tienen a su disposición un número enorme de productos y servicios personalizados, lo quieren todo y lo quieren rápido. Pueden reaccionar inmediatamente a contenidos publicados por una marca y buscar a consumidores con intereses parecidos (Leung, 2013). El poder no sólo se manifiesta a la hora de comprar productos, también buscan empoderarse en su vida y en el trabajo; aspiran a trabajar en industrias más creativas y a estar menos condicionados por estructuras sociales y laborales (Barton, Koslow \& Beauchamp, 2014).

- Poco permeables a la publicidad tradicional. Son más resistentes que las generaciones anteriores a la publicidad y medios tradicionales (Barton, Koslow \& Beauchamp, 2014).

- Críticos y exigentes. Los Milenials son críticos, exigentes y volátiles. Han crecido en una sociedad de consumo, expuestos a las marcas desde su niñez, y son más propensos a considerar a las corporaciones como manipuladoras y agresivas en sus estrategias de comunicación. Con una plétora de productos y servicios a su disposición, un 86 por ciento declara que dejaría de hacer negocios con una empresa debido a una mala 
experiencia de cliente, de modo que las experiencias digitales negativas en línea y móvil tienen un impacto negativo mucho mayor que sobre otros grupos de edad (Estudio BBVA, 2015). Además, buscan la gratificación instantánea, valoran la rapidez, eficacia y conveniencia en todas sus transacciones (Prensky, 2001).

- Rearme ético. Existe consenso en la literatura con que, al menos desde un punto de vista medioambiental y social, los Milenials son más proclives a apoyar causas solidarias. Es una generación que ha crecido en un entorno con problemas medioambientales de gran trascendencia (calentamiento global) y rodeados de una creciente cultura de la sostenibilidad y el reciclaje, por lo que entre sus motivaciones se encuentra la contribución al medio ambiente y el planeta. Apoyan más que generaciones anteriores las causas sociales y a las marcas socialmente responsables (Barton, Koslow \& Beauchamp, 2014).

- Individualismo. A pesar de su alineación con valores éticos y de solidaridad, conforman un grupo donde también conviven valores relacionados con el individualismo, donde el disfrute y el aprovechamiento del momento están por encima de otros valores como la familia, el orden o la tradición (Espinoza et al., 2010).

\subsection{Claves para una estrategia de marca efectiva dirigida a Milenials en Redes Sociales}

La literatura revisada muestra cómo las Redes Sociales han cambiado radicalmente la forma en la que las marcas deben comunicarse con los consumidores. La relación consumidor - marca se ha visto afectada por el poder adquirido por la Generación Y para controlar cómo se comunica con las organizaciones a través de la redes (Duffet, 2015). Los Milenials se relacionan de una forma totalmente distinta con las marcas y han redefinido las formas de comunicarse con las empresas y medios. Según Bellman, Teich y Clark (2009), la Generación Y produce una disrupción que obliga a los profesionales del marketing a redefinir sus estrategias de comunicación, en dónde y cómo alcanzar a su público objetivo, dado que ya no es posible llegar a ellos a través de los medios tradicionales (televisión, radio...) frente a los que las nuevas generaciones se muestra impermeables.

El medio Internet lo ha cambiado todo. La generación Y ve menos televisión, está menos influida por los medios masivos, y es mucho más resistente a los anuncios que las anteriores generaciones. Ahora utiliza una variedad de medios 
que usa regularmente, como Redes Sociales y blogs, a través de los cuales pueden comunicarse y expresar su opiniones y sentimientos (Hershatter \& Epstein, 2010). Los modelos publicitarios clásicos viven tensiones disruptivas que impulsan a las marcas a generar contenidos bajo la fórmula del branded content o contenido de marca, inspirados en las teorías de la narrativa transmediática. La sinergia entre marcas y contenidos que posibilita el branded content comulga con la era postpublicitaria que vivimos, en la que los anunciantes ansían crear relaciones con las personas y hablar su mismo lenguaje (Castelló, Pino \& Tur-Viñes, 2016).

Es notorio que las expectativas de los Milenials son diferentes respecto a generaciones anteriores, y las empresas deben repensar sus marcas, modelos de negocio y marketing adecuadamente (Barton, Koslow \& Beauchamp, 2014). Éstas deben entender las características y valores de esta generación si quieren crear productos y servicios relevantes que satisfagan sus necesidades. Las Redes Sociales permiten a las empresas construir marcas a través de diferentes estrategias de comunicación, principalmente mediante la publicación de contenido que ayude a desarrollar una relación personal con cada cliente. Por lo tanto, esta generación y su presencia en Redes Sociales representa una gran oportunidad para el marketing empresarial, en un entorno donde se han fundido la información, la publicidad y el entretenimiento (Hershatter \& Epstein, 2010). Tener un posicionamiento de marca definido es un elemento fundamental para una exitosa identidad de marca. El propósito o posicionamiento de una marca es la razón principal de ser del negocio, ya que transmite los beneficios que trae la empresa a sus clientes. Hay una gran oportunidad para las empresas para aprovechar las ventajas de la presencia en Redes Sociales y crear un vínculo con sus clientes (Hudson et al., 2015).

Queda claro, por tanto, que las Redes Sociales son el medio a través del cual las marcas deben construir sus relaciones con los clientes, de modo que se logre una buena notoriedad, valor de marca y fidelidad, que son los factores que incluyen en las decisiones de compra (Rohrs, 2014). Sin embargo, la literatura que se ha revisado sobre la Generación Milenial sugiere que hay una brecha en el conocimiento de empresas a la hora de alcanzar a esta generación. Prueba de ello es que muchas empresas y marcas fallan a la hora de construir relaciones con este público a través de los nuevos canales de comunicación, y tienen dificultades en evolucionar desde otros modelos y comportamientos de la publicidad tradicional (Barton, Koslow \& Beauchamp, 2014). Para alcanzar con efectividad a esta generación a través de las Redes Sociales, se debe conocer su comportamiento y motivaciones dentro de las mismas. Lograr usuarios con una alta vinculación con la marca requiere una estrategia en Redes Sociales activa, pero antes de desarrollar los contenidos, la marca 
necesita entender bien a su audiencia para asegurarse de que el contenido es el adecuado (Hudson et al., 2015).

Todo lo expuesto hasta ahora confirma la importancia para las empresas que supone tener una presencia en Redes Sociales para construir marcas a través de diferentes estrategias de comunicación, pero no responde aún a la principal cuestión planteada en este artículo: cuáles son las claves para que las organizaciones logren crear estas marcas para conseguir vínculos fuertes con la Generación Milenial. A continuación se recopilan y sintetizan las principales claves señaladas por los diferentes autores analizados.

De acuerdo con Enginkaya y Yilmaz (2014), las principales motivaciones para interactuar con una marca a través de una red social por parte de un Milenial son: tener un vínculo con la marca, investigación, búsqueda de oportunidades, conversación y entretenimiento. En definitiva, la marca debe ofrecer un contenido entretenido, útil o construir un vínculo emocional, al compartir con el usuario valores, estilo de vida y deseos. Cuando existe este tipo de vínculo, la marca se convierte en una forma de expresión personal y de identidad del Milenial.

Schawbel (2015) señala varios factores clave a tener en cuenta por las marcas que se dirigen a Milenials. Entre ellos, la necesidad de estar presente en diversas plataformas y dispositivos. Además, el mensaje transmitido debe ser auténtico y estar alineado con los valores reales de la marca. Otro factor importante es lograr que el consumidor Milenial participe activamente y se involucre en la comunicación e incluso en la creación de nuevos productos y servicios. El 42 por ciento de los Milenials están interesados en ayudar a las empresas en la creación de nuevos productos y servicios.

La selección de la plataforma más efectiva para alcanzar al Milenial también es un elemento central. Dependerá del sector y del tipo mensaje a transmitir, pero a ser posible se debe tener una presencia multicanal. También es importante que el mensaje y el tono de la comunicación sean relevantes para el público Milenial, y que estén alineados con sus valores y personalidad. La comunicación debe resultar interesante, por lo que se debe abandonar el lenguaje retorico e institucional; el contenido debe entretener, informar y a ser posible estar presentado de forma original. El descubrimiento de la marca a través de prescriptores y el boca a boca son recomendables, y el incorporar testimonios de personas relevantes para el segmento puede ser una estrategia muy efectiva. Un Milenial pregunta a una media de cinco personas de su confianza antes de una compra, comparado con la media de 3 veces que pregunta alguien de la Generación Baby Boomer. La definición de experto para 
un Milenial ya no es una persona con credenciales académicos y profesionales sino alguien que tenga la experiencia de primera mano, idealmente un amigo o alguien cercano. El 48 por ciento de los Milenials aseguran que el boca a boca influye en la decisión de compra más que cualquier anuncio (Barton, Koslow \& Beauchamp, 2014).

El estudio NewsCred Milenial Survey (2014) resalta diferentes aspectos para lograr crear un vínculo entre la marca y el Milenial. Entre ellos destaca el ser original y divertido (el 70 por ciento de los Milenials aseguran que esa es la principal razón para compartirlo), que sea útil (un 64 por ciento se siente vinculado positivamente a una marca que desarrolla un contenido relevante para sus vidas, intereses, deseos y necesidades). La marca debe ser abierta y honesta, explicar claramente qué quiere de su público y qué ofrece exactamente.

Mobolade (2016) hace un amplio repaso a las claves de la comunicación con Milenials en Redes Sociales: el tono de la comunicación debe reflejar los valores y aspiraciones de los Milenials (sinceridad y humildad), pero con cuidado de no tratar de mimetizar de forma artificial el estilo o comunicación joven si una marca no es nativa digital. La oportunidad de tener conversación sincera con la marca representa una motivación importante para los Milenials. La socialización, la interacción y el sentido de comunidad y pertenencia son fundamentales para este tipo de usuario, ya que le permiten tener su propia voz y opinión en relación a la marca. La autenticidad y la simplicidad son valores positivos para esta generación. Lo ideal es lograr que el consumidor hable por la marca, que la prescriba de forma natural, y para esto influye mucho que el Milenial descubra la marca, ya que no le gusta que nada altere su experiencia social. Además, Mobodale apunta a la responsabilidad social como un elemento central; la Generación Y confía más y presta más atención a las empresas con responsabilidad social demostrada.

La comunicación de marca más potente se logra cuando la organización consigue que los Milenials usen la marcas como una forma de expresarse a sí mismos, al sentirse identificados con sus valores. Para los Milenials es especialmente relevante que los valores de la marca sean trascendentales, que contribuyan a cambiar el mundo. Las marcas con valores superiores, que contribuyen a mejorar la sociedad, personas o medioambiente, tienen más posibilidades de conectar con la Generación Y. El 85 por ciento de los consumidores Milenial están influidos por los esfuerzos de responsabilidad social de la marca antes del proceso de compra y antes de recomendarla a otros, y un 73 por ciento está dispuesto a probar una nueva marca si apoya una causa justa (Barton, Koslow \& Beauchamp, 2014). 
Afshar (2016) sitúa al vídeo como uno de los medios más efectivos para alcanzar al público Milenial e interaccionar con la marca, ya que el contenido visual es 12 veces más efectivo que otras formas publicitarias para esta generación. El 60 por ciento de los anuncios digitales tienen contenido audiovisual. El número de vídeos reproducidos en Facebook se duplicó en sólo medio año en 2015. Muchas de las marcas más exitosas expanden el uso del vídeo para buscar conexiones más profundas con el público Milenial. Según Afshar, los cinco tipos de vídeo más efectivos son los testimonios, las demostraciones de producto, los tutoriales, las entrevistas a líderes y los casos de ejemplo.

La transparencia en la comunicación es un factor destacado por muchos autores. Es necesario conectar con una conversación bidireccional para involucrar a la generación Milenial en un proceso comunicativo honesto. Esta generación desea interactuar con las marcas, ser escuchada en cualquier momento y lugar, hablar sobre sus preocupaciones y experiencias. Es necesario construir una conversación sincera y esto implica ceder al consumidor un cierto grado de control, dejarle que establezca los términos de la conversación (Castelló, Pino \& Tur-Viñes, 2016). Las empresas ya no controlan la conversación. Ésta se debe gestionar con cercanía, transparencia e inmediatez para mantener la confianza de sus clientes actuales y no perder clientes potenciales (Burstein, 2013). El 37 por ciento de los Milenials desconfían de las grandes empresas y consultan las Redes Sociales para buscar información y recomendaciones que ellos consideren auténtica. Establecer un diálogo transparente no sólo permite conectar con los usuarios y potenciar así la imagen de marca, además provee a la organización de un contenido auténtico para llenar de valor sus canales sociales, que sirve a su vez para construir la fidelidad hacia la marca a través de esa interacción social (Castelló, Pino \& Tur-Viñes, 2016).

Otros autores inciden en la recompensa como estrategia para atraer a la Generación Milenial. Un segmento dentro de este público interactúa con las marcas en busca de esta recompensa. Dentro de estas motivaciones de tipo más funcional y menos emocional, los beneficios en forma de descuentos o promociones pueden resultar muy efectivos. El 77 por ciento participa en programas de fidelización y el 44 por ciento está dispuesto a promocionar productos y servicios en Redes Sociales a cambio de recompensas. Aún así, las marcas hoy se replantean las estrategias de fidelización y buscan recompensas menos materiales y más experienciales, como el acceso exclusivo a determinado contenido (Schawbel, 2013; Mobolade, 2016). 


\section{Metodología.}

El artículo realiza una recopilación y síntesis de la información más relevante sobre el tema en estudio: las claves de comunicación para generar estrategias de marca efectivas dirigidas a la Generación Milenial a través de las Redes Sociales. Se trata de una construcción del estado del arte mediante investigación cualitativo-documental de carácter crítico-interpretativa que pretende proporcionar al lector una puesta al día sobre conceptos útiles en el área de marca, Redes Sociales y generaciones digitales. La descripción finaliza con un análisis personal del autor y unas recomendaciones a las empresas para que gestionen de manera adecuada su presencia en las Redes Sociales frente al usuario de la Generación Milenial.

Para la localización de los documentos bibliográficos se han utilizado varias fuentes documentales. Se exploraron principalmente artículos publicados en revistas científicas indexadas en las bases de datos Web of Science (WoS), Scopus y Google Scholar. Los descriptores utilizados fueron: Redes Sociales, medios sociales, generación milenial, generación Y, marca, marketing, comunicación. Dada la variedad y dispersión de palabras clave utilizadas en la literatura científica, se trabajó con una búsqueda combinada, en la que se aplicaron conectores booleanos OR y AND.

De manera secundaria, y dado que el tema tratado requiere obtener datos muy recientes, se ha acudido a otras bases de datos, resúmenes, artículos en revistas, así como estudios de grandes corporaciones y consultoras internacionales. 


\section{Resultados y discusión.}

El objetivo del artículo consiste en recopilar qué sabemos hoy sobre las principales claves para generar estrategias de marca efectivas dirigidas a la Generación Milenial a través de las Redes Sociales. La revisión de la bibliografía consultada ha servido para dar respuesta a las tres cuestiones planteadas: ¿Cómo queda delimitada actualmente la Generación Milenial?, ¿Cuáles son las principales características de esta generación? ¿Cuáles son las claves para que las organizaciones logren crear marcas que consigan vínculos fuertes con la Generación Milenial en Redes Sociales?.

En relación a la primera pregunta (¿Cómo queda delimitada hoy la Generación Milenial?), se ha encontrado una literatura extensa que define los límites de esta generación. Estos límites varían ligeramente según la fuente consultada, pero la mayoría de los autores incluyen en ella a las personas nacidas entre los años 1981 y 2000. También se ha podido comprobar cómo el estudio y el interés por esta Generación en el área del marketing aumentaba en los últimos años, conforme los Milenials se hacían adultos. Este grupo ya constituye el principal segmento de consumidores y en pocos años será la gran fuerza laboral del mundo.

En relación a la segunda cuestión, ¿Cuáles son las principales características de esta generación?, se ha comprobado que existe en general un amplio consenso a la hora de definir los atributos que definen a los Milenials, aunque también existen diferentes autores que advierten del riesgo de estereotipar a esta generación. Las principales críticas se refieren al hecho de estudiar a la Generación Y como un grupo único y homogéneo, un enfoque superficial para algunos autores que dificulta establecer estrategias de marketing segmentadas según los diferentes valores, personalidades y estilos de vida que puede haber dentro de toda una generación. A pesar de lo anterior, la mayoría de publicaciones coinciden en la definición de los valores, comportamiento y motivaciones de los Milenials. Estos atributos se pueden resumir en: alta exposición a la tecnología y la información, uso intensivo de las Redes Sociales, comportamiento multiplataforma y multitarea, necesidad de socialización y conexión, empoderamiento frente a organizaciones, baja permeabilidad a los medios publicitarios tradicionales, carácter exigente frente a marcas y empresas, rearme ético frente a causas sociales y medioambientales y personalidad individualista.

En relación a la tercera cuestión, ¿Cuáles son las claves para que las organizaciones creen marcas fuerte en Redes Sociales para Milenials?, la 
bibliografía consultada coincide en que la relación consumidor - marca se ha visto definitivamente afectada por el poder adquirido por la Generación Y a través de las Redes Sociales. Una disrupción que obliga a los profesionales del marketing a redefinir sus estrategias de comunicación. Las Redes Sociales son el medio a través del cual las marcas deben construir sus relaciones con los clientes, de modo que se logre notoriedad, valor de marca y fidelidad. Esta gran oportunidad choca aún con una brecha en el conocimiento de las empresas a la hora de alcanzar a esta generación. Prueba de ello es que muchas empresas y marcas fallan a la hora de construir relaciones con este público a través de los nuevos canales de comunicación. Antes de desarrollar los contenidos, la marca necesita entender bien a su audiencia para asegurarse de que el contenido es el adecuado.

A la hora de recopilar las claves para que las organizaciones logren crear marcas para conseguir vínculos fuertes con la Generación Milenial, el resultado ha sido una literatura muy fragmentada, en la que cada estudio usa una taxonomía propia a la hora de definir las claves de comunicación con esta generación. Aun así, se han encontrado elementos comunes que se han agrupado en diferentes categorías. Éstas son:

1. Comunicación multiplataforma. Dado que el comportamiento de los Milenial es multiplataforma (se mueven indistintamente a través de ellas) las marcas deben analizar qué Redes Sociales son las más adecuadas para la comunicación, y tener en cuenta que puede ser conveniente la presencia en varias de ellas.

2. Lenguaje empático. La Generación Y no acepta el lenguaje retórico e institucional. Es necesario conectar con una conversación bidireccional para involucrar a la generación Milenial en el proceso comunicativo honesto. Esto implica ceder al consumidor un cierto grado de control en la conversación.

3. Contenido relevante. El contenido generado por la marca debe ser relevante para la generación Milenial. Esto implica generar un contenido entretenido, útil o que construya un vínculo emocional con la generación, para compartir con el usuario valores, estilo de vida y deseos.

4. Autenticidad y sinceridad. La autenticidad y la simplicidad son valores importantes para esta generación. El mensaje transmitido debe ser auténtico y estar alineado con los valores reales de la marca, que debe ser abierta, honesta, y explicar claramente qué es, qué quiere de su público y qué ofrece exactamente.

5. Boca a boca / prescripción. La prescripción de terceros y el boca a boca influyen en esta generación más que en las predecesoras. El 
descubrimiento de la marca a través de prescriptores y el boca a boca es altamente recomendable.

6. Valores / personalidad del público. El mensaje y el tono de la comunicación deben estar alineados con los valores y personalidad del Milenial. La comunicación de marca más potente se logra cuando la organización consigue que esta generación use la marca como una forma de expresarse a sí misma, al sentirse identificada con sus valores.

7. Valores superiores de marca. Para los Milenials es especialmente relevante que los valores de la marca sean trascendentales y que contribuyan a cambiar el mundo. Las marcas con valores superiores, que contribuyen a mejorar la sociedad, personas o medioambiente, tienen más posibilidades de conectar con la Generación Y.

8. Participación activa. Conviene lograr que el consumidor Milenial participe activamente y se involucre en la comunicación e incluso en la creación de nuevos productos y servicios de la organización.

9. Contenido audiovisual. El formato vídeo es uno de los medios más efectivos para alcanzar a este público.

10. Recompensa. Un segmento del público Milenial interactúa con las marcas en busca de esta recompensa. Los beneficios en forma de descuentos o promociones pueden resultar muy efectivos.

\section{Conclusiones.}

El artículo ha realizado una recopilación y síntesis de la información más relevante sobre el tema en estudio: las claves de comunicación para generar estrategias de marca efectivas dirigidas a la Generación Milenial a través de las Redes Sociales. Esta revisión pretende proporcionar al lector una puesta al día sobre conceptos útiles en el área de marca, Redes Sociales y generaciones digitales. Las principales conclusiones alcanzadas se pueden resumir en tres puntos:

- La Generación Milenial, que comprende a las personas nacidas entre los años 1981 y 2000, ya constituye el principal segmento de consumidores y en pocos años supondrá la gran fuerza laboral del mundo.

- Los principales atributos que definen y caracterizan a esta generación son: alta exposición a la tecnología y la información, uso intensivo de las Redes Sociales, comportamiento multiplataforma y multitarea, necesidad de socialización y conexión, empoderamiento frente a organizaciones, baja permeabilidad a los medios publicitarios 
tradicionales, carácter exigente frente a marcas y empresas, rearme ético frente a causas sociales y medioambientales y personalidad individualista.

- Las claves de comunicación se pueden resumir en: realizar una comunicación multiplataforma, utilizar un lenguaje empático, desarrollar un contenido relevante, ser auténtico y honesto, utilizar a prescriptores y terceros para que recomienden tu marca, realizar una comunicación alineada con los valores de la generación, tratar de comunicar valores superiores de marca (contribución a una causa superior), fomentar la participación activa (co-crear con los clientes los productos y servicios), desarrollar los contenidos en un formato audiovisual y establecer una estrategia de recompensas.

\section{Bibliografía.}

Afshar, V. (2016). How Video Marketing Will Change the Game for Marketers in 2016. The Huffington Post. Recuperado de http:/ /www.huffingtonpost.com/valaafshar/how-video-marketing-will_b_8962102.html

Alsop, R. (2008). The Trophy Kids Grow Up: How the Milenial Generation is Shaking Up the Workplace. San Francisco: Jossey Bass.

Barbagallo, P. (2003). Teens. Target Marketing, 26(4), 65-68.

Barton, C., Koslow, L., \& Beauchamp, C., (2014). How milenials are changing the face of marketing forever. BCG Perspectives. Recuperado de https://www.bcgperspectives.com/content/articles/marketing_center_consu mer_customer_insight_how_milenials_changing_marketing_forever/

Beauchamp, M. B. \& Barnes, D. C. (2015). Delighting Baby Boomers and Milenials: Factors that matter most. Journal of Marketing Theory and Practice, 23(3), $338-350$.

Bellman, L. , Teich, I. \& Clark, S. (2009), Fashion accessory buying intentions among female millenials, Review of Business, 30(1), 46-57.

Bennett, S., Maton, K. \& Kervin, L. (2008), The 'digital natives' debate: a critical review of the evidence. British Journal of Educational Technology, 39(5), 775-786. 
Bolton, R. N., Parasuraman, A., Hoefnagels, A., Migchels, N., Kabadayi. S., Gruber, T., \& Solnet, D. (2013). Understanding generation Y and their use of social media: A review and research agenda. Journal of Service Management, 24(3), 245-267.

Brosdahl, D. J., \& Carpenter, J.M. (2011). Shopping orientations of US males: a generational cohort comparison. Journal of Retailing and Consumer Services, 18(6), 548-554.

Burstein, D. (2013). Fast Future: How the Milenial Generation is Shaping Our World. Boston: Beacon Press.

Castelló Martínez, A., Del Pino Romero, C. y Tur-Viñes, V. (2016). Estrategias de contenido con famosos en marcas dirigidas a público adolescente. Icono14, 14, 123-154.

DeVaney, S.A. (2015). Understanding the Milenial Generation. Journal of Financial Service Professionals, 69(6), 11-14.

Duffett, R. G. (2015). Facebook advertising's influence on intention-topurchase and purchase amongst Milenials. Internet Research, 25(4), 498-526.

Enginkaya, E., \& Y1lmaz, H. (2014). What drives consumers to interact with brands through social media? A motivation scale development study. Procedia Social and Behavioral Sciences, 148(0), 219-226.

Espinoza, C., Ukleja, M., \& Rusch, C. (2010). Managing the Milenials: Discover the core competencies for managing today's workforce. Hoboken, NJ: John Wiley and Sons.

Estudio BBVA (2015). Generación Milenial: así es la primera generación completamente digital. Centro de Innovación $B B V A$. Recuperado de https://www.centrodeinnovacionbbva.com/sites/default/files/ebook-cibbvainnovation-trends-generacion-milenials.pdf

Hershatter, A. \& Epstein, M. (2010). Millenials and the world of work: an organization and management perspective". Journal of Business Psychology, 25(2), 211-223.

Hudson, S., Huang, L., Roth, M. S., \& Madden, T. J. (2015). The influence of social media on consumer-brand relationships: A three-country study of brand perceptions and marketing behaviours. International Journal of Research in Marketing, 33(2016), 27-41. 
Hyllegard, K., Yan, R., Ogle, J. \& Attmann, J. (2011). The influence of gender, social cause, charitable support, and message appeal on Gen Y's responses to cause-related marketing. Journal of Marketing Management, 27(1-2), 100-123.

Leung, L. (2013). Impacts of net-generation attributes, seductive properties of the internet, and gratificationsobtained on internet use. Telematics and Informatics, 20(2), 107-129.

Mobolade, O. (2016). How to market effectively to Millennials. Recuperado de https://www.warc.com/SubscriberContent/Article/A107430_How_to_mark et_effectively_to_Millennials/107430

NewsCred Milenial Survey (2014). Newscreed. Recuperado de http://www.newscred.com/wp-

content/themes/newscred/assets/downloads/guide/NewsCred_Milenial_Mi nd.pdf

Parment, A. (2011). Generation Y in Consumer and Labour Markets. New York; Routledge.

Prensky, Marc. (2001). Digital Natives, Digital Immigrants. On the Horizon, 9(5), 1-6.

Rohrs, J.K. (2014). Audience: Marketing in the Age of Subscribers, Fans \& Followers. Hoboken, NJ: John Wiley \& Sons.

Schawbel, D. (2013). 74 Of The Most Interesting Facts About Milenials. Dan Schawbel. Recuperado de http://danschawbel.com/blog/74-of-the-mostinteresting-facts-about-the-milenial-generation/

Smith, K.T. (2012). Longitudinal study of digital marketing strategies targeting Milenials. The Journal of Consumer Marketing, 29(2), 86-92.

Statista (2016). Number of global social network users 2010-2020. Recuperado de http://www.statista.com/statistics/278414/number-of-worldwide-socialnetwork-users /

Swinarski, M., Parente, D. \& Noce, K. (2010). A study of gender differences with respect to internet socialization of adolescents. Journal of Business \& Economics Research, 8(6), 23-30. 
Syrett, M. \& Lammiman, J. (2003). Catch them if you can. Director, 57(3), 7076.

Tuten,T. \& Solomon, M. (2014). Social Media Marketing. Harlow: Pearson.

Twenge, J. M. (2009). Generational changes and their impact in the classroom: Teaching generation me. Medical Education, 43(5), 398-405.

Wesner, M.S. \& Miller, T. (2008). Boomers and Millenials have much in common. Organizational Development, 26(3), 89-96. 


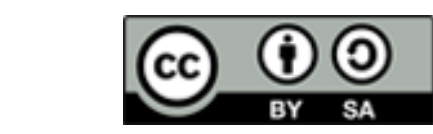

Licencia Creative Commons

Miguel Hernández Communication Journal

mhjournal.org

\section{Cómo citar este texto:}

José Javier Ruiz Cartagena (2017): "Millennials y redes sociales: estrategias para una comunicación de marca efectiva", en Miguel Hernández Communication Journal, nº, pp. 347 a 367. Universidad Miguel Hernández, UMH (Elche-Alicante). Recuperado el — de de 20__ de: Џink del artículo en mhjournal.org] 
